\title{
Photon emission by volume reflected electrons in bent crystals
}

\author{
C. F. Nielsen $\odot,{ }^{1}$ U. I. Uggerh $ø j \odot,{ }^{1}$ R. Holtzapple $\odot,{ }^{2}$ T. W. Markiewicz, ${ }^{3}$ B. C. Benson, ${ }^{3}$ \\ E. Bagli, ${ }^{4}$ L. Bandiera, ${ }^{4}$ V. Guidi, ${ }^{4}$ A. Mazzolari, ${ }^{4}$ and U. Wienands ${ }^{5}$ \\ ${ }^{1}$ Department of Physics and Astronomy, Aarhus University, 8000 Aarhus C., Denmark \\ ${ }^{2}$ Department of Physics, California Polytechnic State University, San Luis Obispo, California 93407, USA \\ ${ }^{3}$ SLAC National Accelerator Laboratory, 2575 Sand Hill Road, Menlo Park, California 94025, USA \\ ${ }^{4}$ Department of Physics and Earth Sciences of the University of Ferrara, and INFN Section of Ferrara, \\ Via Saragat 1/C, I-44122 Ferrara, Italy \\ ${ }^{5}$ Argonne National Laboratory, 9700 S. Cass Avenue, Lemont, Illinois 60439, USA
}

(Received 17 September 2019; published 15 November 2019)

\begin{abstract}
We present a quantitative experimental analysis of the radiation emission of $12.6 \mathrm{GeV}$ electrons moving along and across the (111) planes of a strongly bent silicon crystal with a bending radius of $0.15 \mathrm{~m}$. We have measured the radiation emitted during volume reflection, which has shown to strongly enhance the emission of low-energy photons, and a convincing agreement between simulations and experimental data is found.
\end{abstract}

DOI: 10.1103/PhysRevAccelBeams.22.114701

\section{INTRODUCTION}

Radiation from electrons and positrons channeled in straight oriented single crystals has been studied in great detail over the past decades [1,2]. These studies have shown that radiation emission is enhanced considerably due to the coherent motion and the strong electric fields, which are of the order $10^{11}-10^{13} \mathrm{~V} / \mathrm{m}$ depending on the crystal material, orientation and lattice type. In this paper we study the radiation emitted from electrons during penetration of a strongly bent planar oriented Si crystal. The motion of charged particles penetrating strongly bent crystals has been studied in great detail [3-7], but with little experimental emphasis on their radiation emission. These studies show that particles undergo several different processes during penetration of a bent crystal: mainly channeling, curvature or multiple scattering dechanneling, volume capture (VC) and volume reflection (VR). Here, in particular, we are interested in the radiation emitted during the process of VR. During VR the electrons are exposed, for a prolonged time, compared to the oscillatory motion when channeling or skipping the crystal planes, to a strong part of the electric field in the crystal, which leads to an enhancement of radiation emission which we show is almost synchrotron like. As we show below, the radiation from the VR process can indeed be identified, and our experiment agrees well with numerical VR simulations. In our

Published by the American Physical Society under the terms of the Creative Commons Attribution 4.0 International license. Further distribution of this work must maintain attribution to the author(s) and the published article's title, journal citation, and DOI. experiment we measure the single photon spectrum from 12.6 GeV electrons passing nearly parallel to the (111) planes of a $60 \mu \mathrm{m}$ bent silicon crystal with a bending radius of $0.15 \mathrm{~m}$. By rotating the crystal we vary the incident angle of the electrons hitting the crystal, which enables a study of the radiation emitted from the different processes during passage through the crystal.

This experiment serves as a more detailed study of the nature of VR radiation than previous studies, see: [8-11], which where all made at significantly higher and lower energies than this experiment.

\section{EXPERIMENT}

The experiment was conducted at the SLAC National Accelerator Laboratory End Station A Test Beam (ESTB) facility. The experimental setup is shown in Fig. 1. The incoming secondary electron beam rate is $5 \mathrm{~Hz}$ with an average of 10 electrons as measured by exposing the counter to the beam and analyzing the resulting spectrum in terms of a Poisson distribution. The secondary beam has an estimated energy spread of a few percent and divergence of $75 \mu \mathrm{rad}$. The crystal is mounted on a horizontally translating stage and horizontally rotating stage, capable of rotating in $\simeq 5 \mu \mathrm{rad}$ steps. The vertical bending magnet removes the electrons from the beam line after the crystal, dumping them in the collimators which consists of $25 \mathrm{~cm}$ $\mathrm{Cu}$ followed by $60 \mathrm{~cm}$ of $\mathrm{Pb}$, ensuring that only photons produced in the crystal hit a scintillating fiber (SciFi) calorimeter positioned approx. $48 \mathrm{~m}$ downstream of the crystal. The SciFi has an energy resolution of around $10 \%$ for the photon energies of relevance here. The SciFi has a detection surface area of $9 \times 9 \mathrm{~cm}^{2}$ and is $30 \mathrm{~cm}$ deep, 


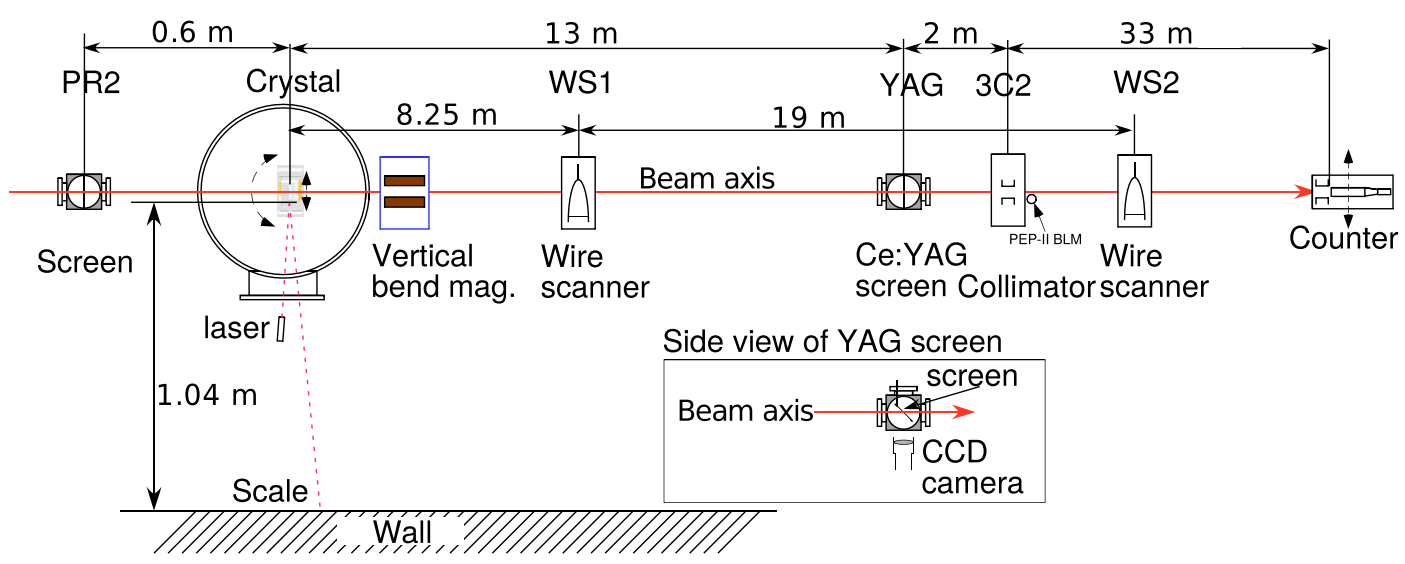

FIG. 1. Sketch of the experimental setup at the SLAC ESTB. Distances are not to scale.

which is large enough to minimize shower leakage. Each electron hitting the $60 \mu \mathrm{m}$ crystal produces on average 0.025 photons; the calculated average pile-up rate for 0.25 photons/pulse is about $6 \%$.

To find the plane of the crystal, the primary electron beam with a divergence of $\sigma \approx 5 \mu \mathrm{rad}$ and $10^{8}$ electrons per pulse was used. By imaging a Cerium doped Yttrium Aluminium Garnet (YAG) screen of $500 \mu \mathrm{m}$ thickness, placed in the primary beam after the crystal, we are able to determine when the beam hits the crystal parallel to the plane, as a portion of the beam is deflected due to channeling through the bent crystal. On Fig. 2 an image of the YAG screen is shown for the aligned orientation where the beam hits the crystal parallel to the plane at the crystal entrance face. The almost circular light peak to the right in the image originates from the channeled particles, near which the so-called quasi-channeling oscillations can be seen [12], and to the left is seen the volume reflected particles and/or the fraction of particles that is not channeled due to the finite beam divergence and its surface

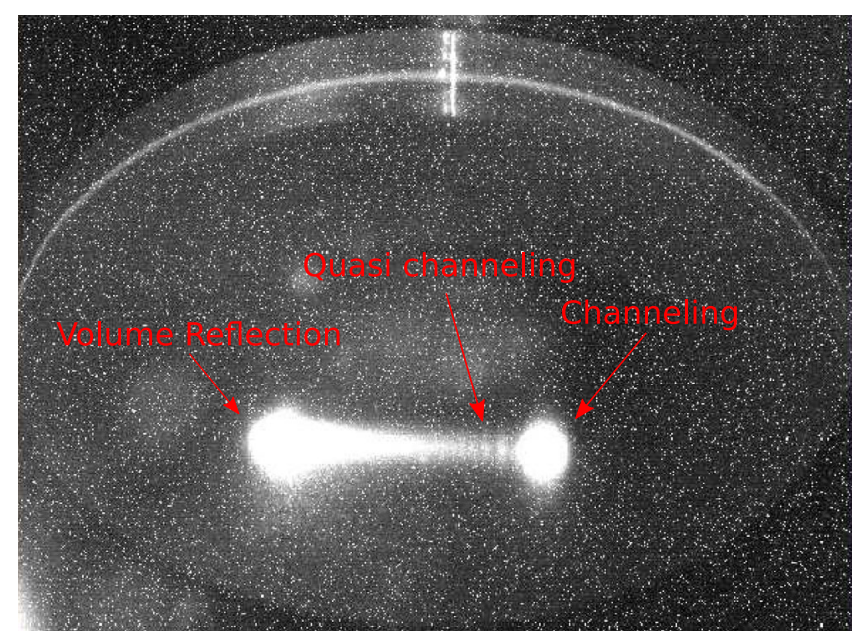

FIG. 2. Image of the YAG screen with the crystal in channeling orientation. transmission. After identifying the direction of the plane, we switched to the secondary beam which has a significantly higher divergence of around $\sigma \approx 75 \mu \mathrm{rad}$. To set the average number of electrons per pulse to the desired value of 10 , the collimators were gradually opened until the SciFi spectrum matched the precalculated shape and energy range, followed by repowering the bending magnet to allow for radiation measurements.

A background spectrum with the crystal moved out of the beam is measured and subtracted from every experimental spectrum measured with a crystal in the beam. The background photons mainly originate from bremsstrahlung emitted from electrons hitting objects along the beam-line. Per linac pulse, there is about the same number of background photons present as coming from the crystal in amorphous orientation, for a signal-to-noise ratio of $1: 1$ in this case.

To compare simulated radiation spectra with the experimental spectra, the efficiency of the setup must be known. Our data in amorphous orientation of the crystal agrees with the expected Bethe-Heitler distribution indicating that the counter efficiency does not depend on the photon energy in our spectral range. Therefore we can find an efficiency factor by measuring the amorphous spectrum from electrons traversing the crystal at an angle far from any low-index crystallographic orientation. Then by normalizing the analytical Bethe-Heitler bremsstrahlung spectrum [13] to the experimentally obtained spectrum, we find the efficiency factor of the setup. Every simulated spectrum compared to experimental spectra should then be multiplied by this factor.

\section{THEORETICAL SIMULATION}

In our theoretical simulation we consider electrons with an energy of $12.6 \mathrm{GeV}$, moving on a trajectory determined by classical electrodynamics, in the electric field of the thermally averaged Doyle-Turner potential [14]. We will be considering the (111) plane of a silicon crystal, bent with a bending radius of $0.15 \mathrm{~m}$ as in the experiment. 
Our simulation code has been scrutinized against some of our previous results for particle deflection with bent crystals [5,15], and reproduces earlier observations. With a classical trajectory we can determine the radiation emitted throughout the crystal as described in [16], variations of which has been employed in recent studies $[17,18]$ investigating the effect of radiation reaction on the emission spectrum of positrons and electrons. We use the Belkacem, Cue and Kimball (BCK) radiation integral $[17,19,20]$ to evaluate the theoretical radiation spectra from the numerically simulated trajectories. The BCK model is a semiclassical model which takes into account the photon recoil and the spin of the particle when evaluating the radiation spectrum. This gives us the radiated photon intensity spectrum of a single electron following a particular trajectory, but since multiple Coulomb scattering has a large effect on dechanneling, and therefore the radiation spectrum, the radiation spectra are averaged over many particles with identical initial conditions. To also account for the beam divergence in the experiment, the initial angle of the electrons is randomly sampled from a Gaussian distribution with mean zero and $\sigma=75 \mu \mathrm{rad}$. When an angular distribution of the particles are used, the initial conditions of each particle is different, so the number of particles used in a simulation is chosen to ensure convergence of the resulting averaged spectra, this number is around 1000 particles for this experiment.

Since the electrons only undergo VR once, and in a short section of the crystal, the overall spectrum from the entire crystal is dominated by coherent bremsstrahlung. To see the radiation spectrum pertaining to VR we divide the crystal into sections and evaluate the radiation spectrum for each section. In Figs. 3 and 4 the velocity perpendicular to the crystal plane as a function of distance traversed in the crystal and the radiation spectrum from each $10 \mu \mathrm{m}$ crystal section, respectively, is shown for a single electron hitting the crystal at an entry angle of $175 \mu \mathrm{rad}$. In section 3 the electron undergoes VR where its simulated spectrum clearly shows that the radiation pertaining to the VR process has a large enhancement in low-energy photon emission. The spectrum in Fig. 4 is from only one electron and does not include multiple Coulomb scattering. Since almost the entirety of section 3 is acceleration in the same direction, the radiation spectrum should be synchrotron like. On Fig. 4 the synchrotron spectrum [21] for a 12.6 GeV electron moving on a curved trajectory with a bending radius of $\rho=0.03 \mathrm{~m}$ is shown, which is a compromise between the average bending radius for this section $\rho \approx 0.06 \mathrm{~m}$ and the smallest bending radius experienced in the VR process, $\rho=0.02 \mathrm{~m}$. The synchrotron spectrum is properly scaled according to a trajectory of the same length as the VR part of section 3 shown on Fig. 3. At an average bending radius of $0.03 \mathrm{~m}$ during the reflection process, the bending takes place on the order of 1000 atomic distances, which justifies the use of the

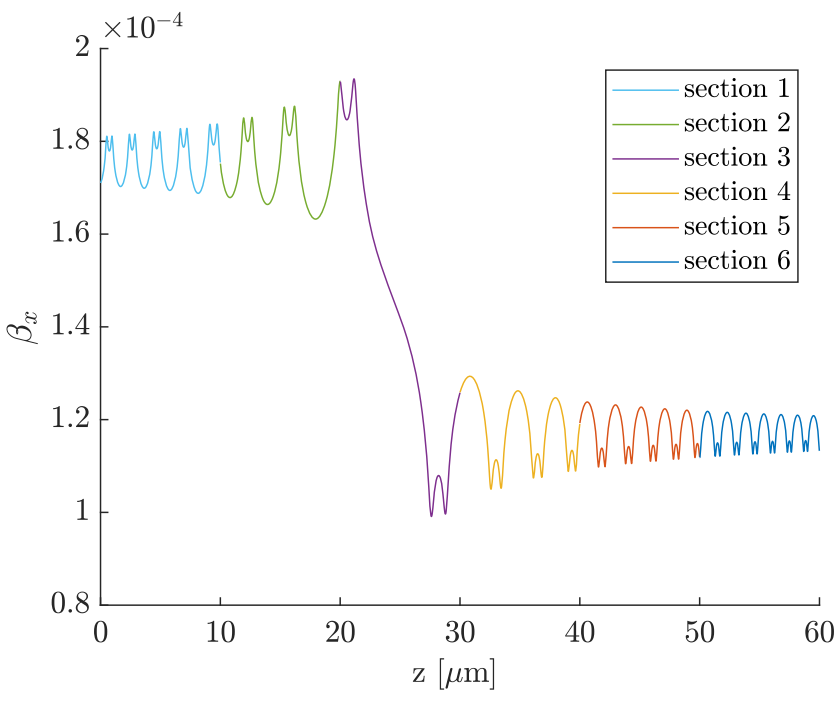

FIG. 3. Reduced velocity $\beta_{x}=v_{x} / c$ perpendicular to the plane as a function of distance traveled in the crystal, for a $12.6 \mathrm{GeV}$ electron hitting the (111) plane of a bent silicon crystal with a bending radius of $0.15 \mathrm{~m}$ and an entry angle of $175 \mu \mathrm{rad}$. Here multiple Coulomb scattering is not included. Each color represents a section in which the radiation spectrum has been evaluated. The corresponding spectra are shown in Fig. 4.

continuum model in analyzing the VR process. This being so, it can be argued that the description of the relevant part of the VR spectrum as a form of synchrotron radiation is meaningful in this context. A synchrotron like spectrum is

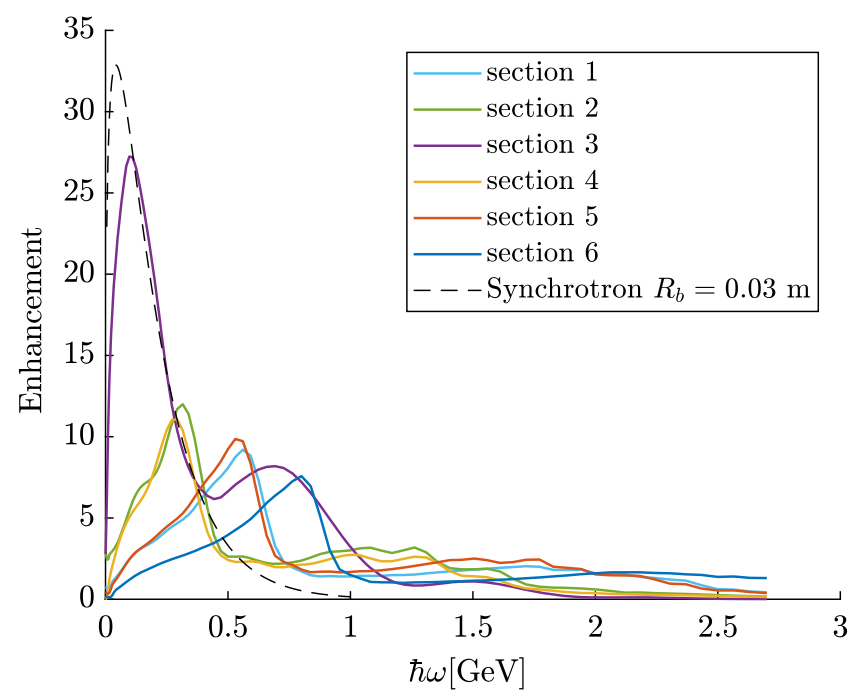

FIG. 4. Radiation spectra from a $12.6 \mathrm{GeV}$ electron hitting the (111) plane of a bent silicon crystal with a bending radius of $0.15 \mathrm{~m}$ and an entry angle of $175 \mu \mathrm{rad}$. Here multiple Coulomb scattering is not included. The color of each spectrum pertains to the respective section in Fig. 3 with the same color. The black dashed curve is the synchrotron spectrum for a $12.6 \mathrm{GeV}$ electron with a characteristic frequency of $\hbar \omega_{c}=0.3 \mathrm{GeV}$, which corresponds to a bending radius of $0.03 \mathrm{~m}$. 


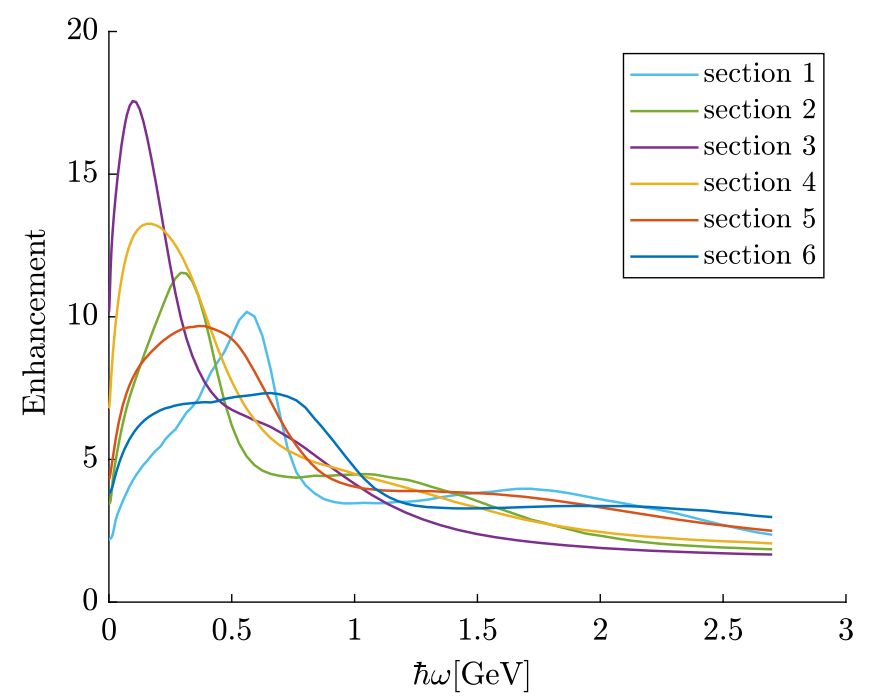

FIG. 5. Radiation spectra from $100012.6 \mathrm{GeV}$ electrons hitting the (111) plane of a bent silicon crystal with a bending radius of $0.15 \mathrm{~m}$ and an entry angle of $175 \mu \mathrm{rad}$ including multiple scattering. The color of each spectrum pertains to its respective $10 \mu \mathrm{m}$ section throughout the crystal as given in Fig. 3 .
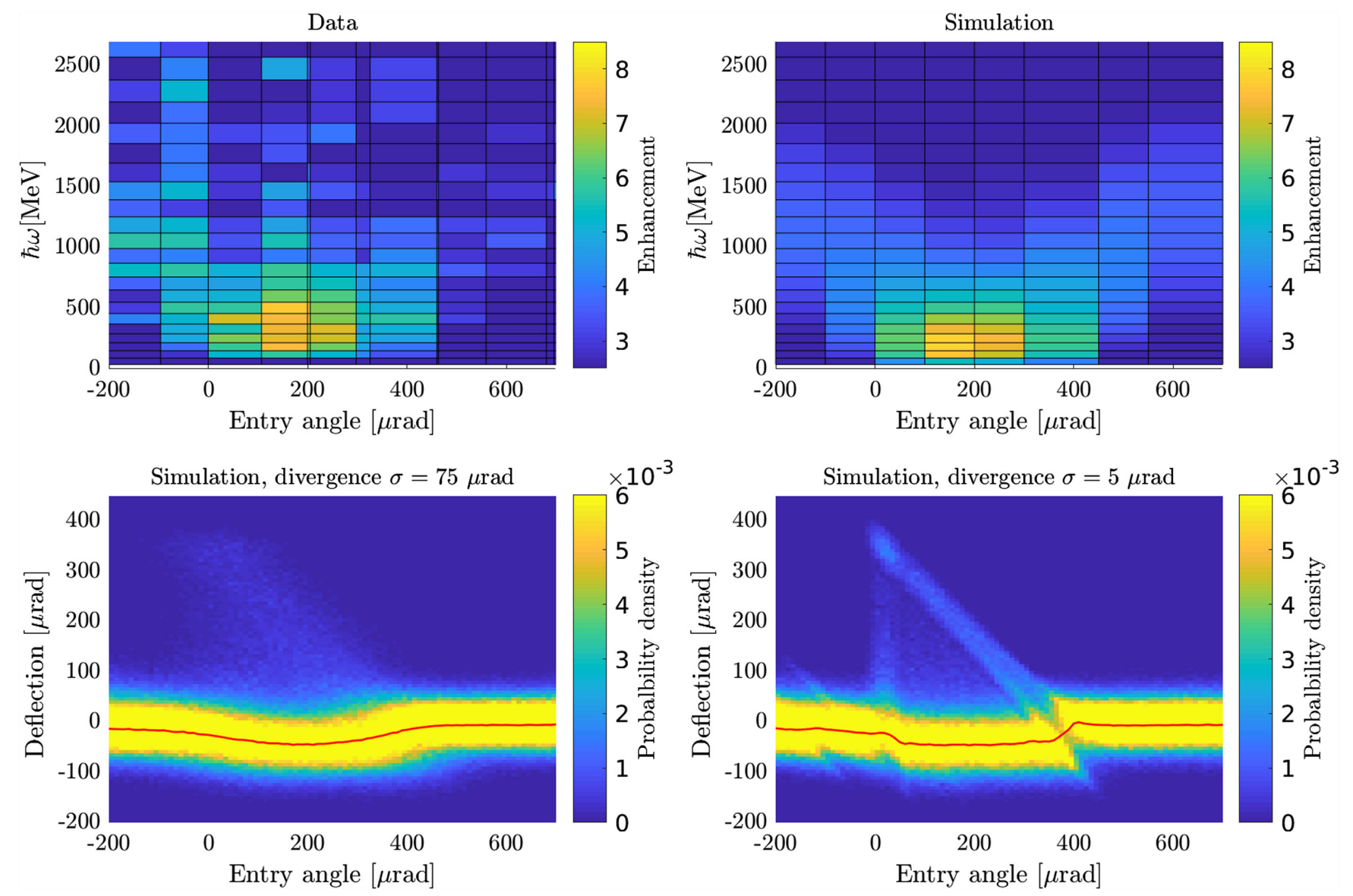

also measured in [22], which could be due to VR, but no estimate was made on how much of the spectrum pertain to VR.

In Fig. 5 the spectra for each section is shown determined by averaging for 1000 independent electrons with an entry angle of $175 \mu \mathrm{rad}$ and multiple scattering included. It is evident that when multiple scattering is included, radiation from VR is still clearly visible in section 3 but also visible in section 4 because particles now also undergo VR in section 4 due to multiple scattering. The sharp features of the spectra seen in Fig. 4 are in Fig. 5 smeared due to multiple scattering when averaging over many particles with slightly different trajectories.

Since the radiation before and after VR is so similar (see Figs. 4 and 5), even with differences imposed by multiple scattering, we expect to see fairly similar spectra for particles with varying entry angles between 100-300 $\mu \mathrm{rad}$.

\section{RESULTS AND DISCUSSION}

The measurement program featured 9 runs, each with a different entry angle with respect to the crystal plane.

FIG. 6. Radiation and deflection spectra from $12.6 \mathrm{GeV}$ electrons with different entry angles with respect to the (111) plane of a $60 \mu \mathrm{m}$ thick bent silicon crystal. The top left figure is the experimental radiation spectra and the top right figure is the simulated radiation spectra for electron beams with a beam divergence of $\sigma=75 \mu \mathrm{rad}$. The bottom figures are the simulated angular deflection of electrons for beams with different entry angles and beam divergences. The red line is the centroid of the lower ridge. 

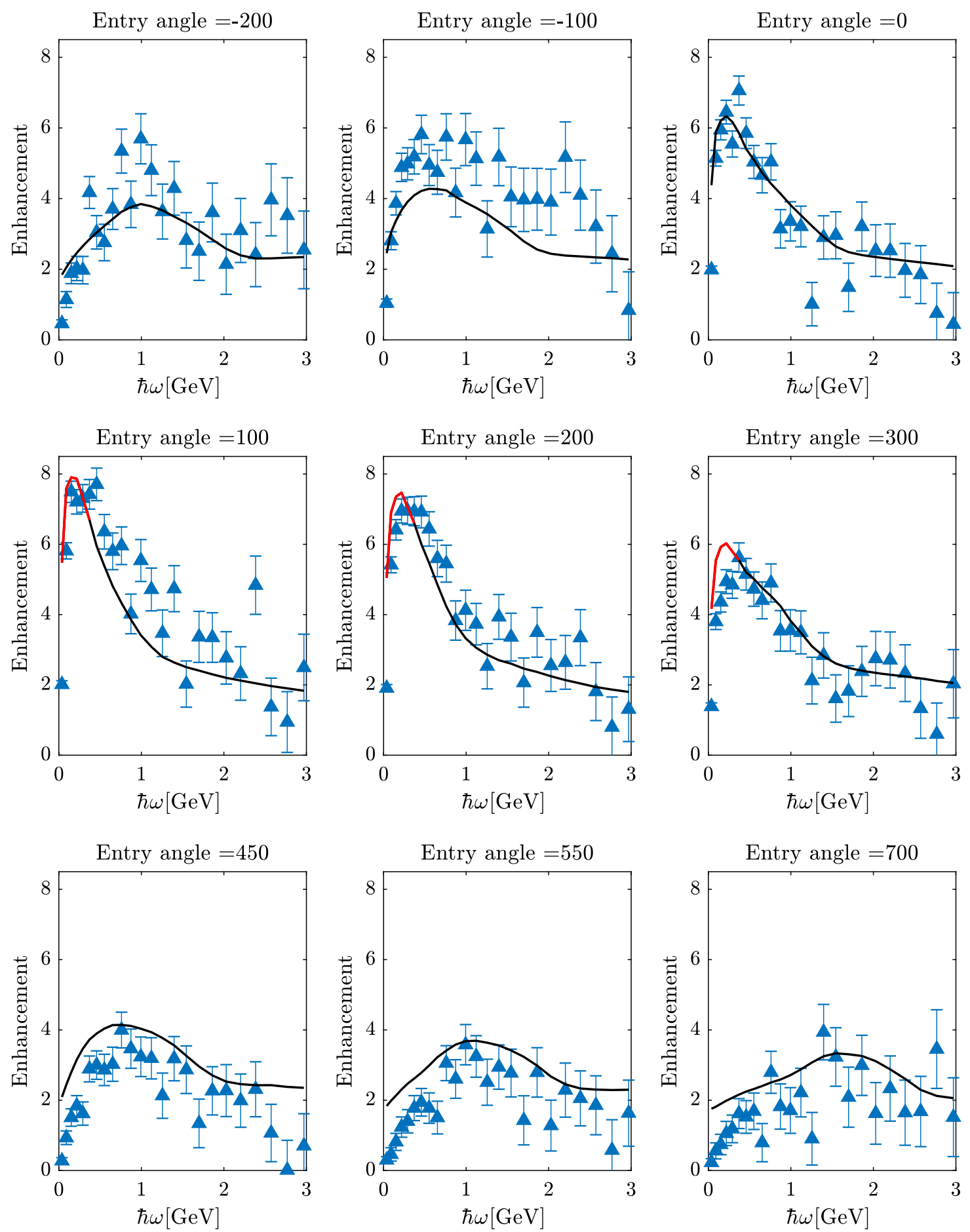

FIG. 7. Radiation spectra from $12.6 \mathrm{GeV}$ electrons with different entry angles with respect to the (111) plane of a $60 \mu \mathrm{m}$ thick bent silicon crystal. The blue data points are the experimental data points and the black curves show the results of the theoretical simulation which has no free parameters. The red curve highlights the area of the spectrum which is dominated by VR as seen from Figs. 4 and 5 .

The different average beam entry angles, listed in $\mu \mathrm{rad}$, are $\theta_{0}=[-200,-100,0,100,200,300,450,550,700]$, where 0 corresponds to the electron beam being aligned parallel to the crystal plane on entry. In Fig. 6, the upper figures are the measured and simulated radiation spectra as a function of entry angle where the simulated electron beam has a beam divergence of $\sigma=75 \mu \mathrm{rad}$. The lower figures are simulated deflection plots at the beam divergences of $\sigma=5 \mu \mathrm{rad}$ and $\sigma=75 \mu \mathrm{rad}$. Several conclusions can be made from the experimental and simulated results: (i) Electrons with zero deflection have passed the crystal without undergoing VR or VC at any point within the 
crystal, while electrons with a considerable negative deflection have undergone VR at some point inside the crystal. (ii) Electrons with positive deflection have at some point been channeled within the crystal, and have either channeled through the rest of the crystal after undergoing VC (the hypotenuse of the triangle) or have been dechanneled after at some point channeling along the planes (body of triangle). (iii) The red line follows the centroid of the lower ridge, which shows that the majority of particles within 100-300 $\mu \mathrm{rad}$ undergo VR. The reason we show the $\sigma=5 \mu \mathrm{rad}$ deflection plot is to illustrate the features of the triangle more clearly. With $\sigma=75 \mu \mathrm{rad}$, as is relevant for the experiment, these features are still visible but not very clear. (iv) By looking at the deflections in this plot we observe that the majority of the electrons with incidence angles between $\simeq 100 \mu \mathrm{rad}$ and $\simeq 350 \mu \mathrm{rad}$ undergo $\mathrm{VR}$ which means that the radiation spectra should have signs of the corresponding radiation.

Comparing the measured and the simulated radiation spectra in Fig. 6, there is good qualitative agreement. Even the features of the spectrum at larger crystal angles - the "wings" due to coherent bremstrahlung-are visible in both spectra. A more quantitative comparison is seen in Fig. 7 where each experimental spectrum corresponding to a certain mean entry angle is shown together with their respective theoretical spectrum. Also here the features of the spectra are in good agreement. The reader is reminded that only one overall efficiency factor is used in this comparison; i.e., there are no fitting parameters introduced in the simulation.

Looking at the spectra between 100-300 $\mu$ rad entry angle, we see a convincing agreement between the experimental values and the theoretical simulation. The enhancement in radiation intensity for low-energy photons, which is an enhancement of about 8 at $0.2 \mathrm{GeV}$ with a standard deviation of $0.25 \mathrm{GeV}$, is clear in this area, and from the discussion earlier, this enhancement should be, and we conclude is, the effect of VR. The red lines in Fig. 7 highlight the part of the spectrum where VR dominates, as seen in Figs. 4 and 5.

The offset on the simulated spectra in the range $450-700 \mu \mathrm{rad}$ we believe is due to statistical errors regarding the normalization of the simulated spectrum. With the large distance between the crystal and the SciFi, which has a $9 \times 9 \mathrm{~cm}$ detection surface, the acceptance depends strongly on the opening angle of the photons, which changes significantly due to the bending of the electron trajectory in the crystal.

\section{ACKNOWLEDGMENTS}

The numerical results presented in this work were partly obtained at the Centre for Scientific Computing, Aarhus http://phys.au.dk/forskning/cscaa and by support from Nvidias GPU grant program. This work was partially supported by the U.S. National Science Foundation
(Grant No. PHY-1535696) and by the U.S. DOE under Contract No. DE-AC02-76SF00515.

[1] U. I. Uggerh $\varnothing \mathbf{j}$, The interaction of relativistic particles with strong crystalline fields, Rev. Mod. Phys. 77, 1131 (2005).

[2] A.H. Sørensen, Channeling, bremsstrahlung and pair creation in single crystals, Nucl. Instrum. Methods Phys. Res., Sect. B 119, 2 (1996).

[3] A. Baurichter et al., Channeling of high-energy particles in bent crystals-Experiments at the CERN SPS, Nucl. Instrum. Methods Phys. Res., Sect. B 164-165, 27 (2000).

[4] W. Scandale et al., Deflection of $400 \mathrm{GeV} / \mathrm{c}$ proton beam with bent silicon crystals at the CERN Super Proton Synchrotron, Phys. Rev. ST Accel. Beams 11, 063501 (2008).

[5] T. N. Wistisen, U. I. Uggerhøj, U. Wienands, T.W. Markiewicz, R. J. Noble, B. C. Benson, T. Smith, E. Bagli, L. Bandiera, G. Germogli, V. Guidi, A. Mazzolari, R. Holtzapple, and S. Tucker, Channeling, volume reflection, and volume capture study of electrons in a bent silicon crystal, Phys. Rev. Accel. Beams 19, 071001 (2016).

[6] J. Forster, H. Hatton, R. Toone, G. Este, S. Baker, R. Carrigan, W. Gibson, R. Wijayawardana, J. Ellison, L. Emman-Wori, and B. Kolbesen, Deflection of GeV particle beams by channeling in bent crystal planes of constant curvature, Nucl. Phys. B318, 301 (1989).

[7] V. Biryukov, Y. Chesnokov, and V. Kotov, Crystal Channeling and Its Application at High-Energy Accelerators (Springer-Verlag, Berlin Heidelberg New York, 1969).

[8] L. Bandiera, E. Bagli, V. Guidi, A. Mazzolari, A. Berra, D. Lietti, M. Prest, E. Vallazza, D. De Salvador, and V. Tikhomirov, Broad and Intense Radiation Accompanying Multiple Volume Reflection of Ultrarelativistic Electrons in a Bent Crystal, Phys. Rev. Lett. 111, 255502 (2013).

[9] V. Guidi, L. Bandiera, and V. Tikhomirov, Radiation generated by single and multiple volume reflection of ultrarelativistic electrons and positrons in bent crystals, Phys. Rev. A 86, 042903 (2012).

[10] W. Scandale et al., Experimental study of the radiation emitted by $180-\mathrm{GeV} / \mathrm{c}$ electrons and positrons volume-reflected in a bent crystal, Phys. Rev. A 79, 012903 (2009).

[11] L. Bandiera, E. Bagli, G. Germogli, V. Guidi, A. Mazzolari, H. Backe, W. Lauth, A. Berra, D. Lietti, M. Prest, D. De Salvador, E. Vallazza, and V. Tikhomirov, Investigation of the Electromagnetic Radiation Emitted by Sub-GeV Electrons in a Bent Crystal, Phys. Rev. Lett. 115, 025504 (2015).

[12] T. N. Wistisen, R. E. Mikkelsen, U. I. Uggerhøj, U. Wienands, T. W. Markiewicz, S. Gessner, M. J. Hogan, R. J. Noble, R. Holtzapple, S. Tucker, V. Guidi, A. Mazzolari, E. Bagli, L. Bandiera, and A. Sytov (SLAC E-212 Collaboration), Observation of Quasichanneling Oscillations, Phys. Rev. Lett. 119, 024801 (2017).

[13] M. Tanabashi et al. (Particle Data Group), Review of Particle Physics, Phys. Rev. D 98, 030001 (2018). 
[14] P. A. Doyle and P. S. Turner, Acta Crystallogr. Sect. A 24, 390 (1968).

[15] U. Wienands, T. W. Markiewicz, J. Nelson, R. J. Noble, J. L. Turner, U. I. Uggerhøj, T. N. Wistisen, E. Bagli, L. Bandiera, G. Germogli, V. Guidi, A. Mazzolari, R. Holtzapple, and M. Miller, Observation of Deflection of a Beam of Multi-GeV Electrons by a Thin Crystal, Phys. Rev. Lett. 114, 074801 (2015).

[16] C. F. Nielsen, Gpu accelerated simulation of channeling radiation of relativistic particles, arXiv:1910.10391.

[17] C. F. Nielsen, J. B. Justesen, A. H. Sørensen, R. Holtzapple, and U. I. Uggerhøj, Experimental verification of the LandauLifshitz equation (to be published).

[18] T. N. Wistisen, A. Di Piazza, C. F. Nielsen, A. H. Sørensen, and U. I. Uggerhøj (CERN NA63 Collaboration), Quantum radiation reaction in aligned crystals beyond the local constant field approximation, Phys. Rev. Research 1, 033014 (2019).

[19] A. Belkacem, N. Cue, and J. Kimball, Theory of crystalassisted radiation and pair creation for imperfect alignment, Phys. Lett. A 111, 86 (1985).

[20] J. Kimball, N. Cue, and A. Belkacem, Crystal-assisted quantum electrodynamics; pair production and radiation, Nucl. Instrum. Methods Phys. Res., Sect. B 13, 1 (1986).

[21] J.D. Jackson, Classical Electrodynamics, 3rd ed. (John Wiley and Sons, New York, 1998).

[22] H. Backe, D. Krambrich, W. Lauth, K. Andersen, J. L. Hansen, and U. I. Uggerhøj, Radiation emission at channeling of electrons in a strained layer $S i_{1-x} G e_{x}$ undulator crystal, Nucl. Instrum. Methods Phys. Res., Sect. B 309, 37 (2013). 